\title{
Hereditarily indecomposable inverse limits of graphs
}

\author{
by \\ K. Kawamura (Tsukuba), H. M. Tuncali (North Bay) \\ and E. D. Tymchatyn (Saskatoon)
}

\begin{abstract}
We prove the following theorem: Let $G$ be a compact connected graph and let $f: G \rightarrow G$ be a piecewise linear surjection which satisfies the following condition: for each nondegenerate subcontinuum $A$ of $G$, there is a positive integer $n$ such that $f^{n}(A)=G$. Then, for each $\varepsilon>0$, there is a map $f_{\varepsilon}: G \rightarrow G$ which is $\varepsilon$-close to $f$ such that the inverse limit $\left(G, f_{\varepsilon}\right)$ is hereditarily indecomposable.
\end{abstract}

1. Introduction. Throughout the paper, a graph means a compact, connected, one-dimensional polyhedron. A continuum (i.e. a compact, connected, metric space) is said to be indecomposable if it is not the union of two proper subcontinua. A continuum is said to be hereditarily indecomposable if each subcontinuum is indecomposable. For a continuous surjection $f: X \rightarrow X$ of a compact metric space $X,(X, f)$ denotes the inverse limit of the inverse sequence

$$
X \stackrel{f}{\leftarrow} X \stackrel{f}{\leftarrow} X \stackrel{f}{\leftarrow} X \stackrel{f}{\leftarrow} \cdots
$$

where all coordinate spaces are $X$ 's and all bonding maps are f's. In this paper, we are concerned with the following question:

What properties of a map $h: G \rightarrow G$ of a graph $G$ imply that for each $\varepsilon>0$, there is a map $h_{\varepsilon}: G \rightarrow G$ which is $\varepsilon$-close to h such that $\left(G, h_{\varepsilon}\right)$ is hereditarily indecomposable?

Properties of maps on graphs which yield inverse limits containing indecomposable subcontinua have been investigated by several authors. In particular, Barge and Diamond [2] obtained necessary and sufficient conditions for piecewise monotone maps whose inverse limits contain indecomposable

2000 Mathematics Subject Classification: 54F15, 54H20.

Key words and phrases: graph, hereditarily indecomposable, inverse limit.

Part of this research has been done while the first named author was visiting the University of Saskatchewan on NSERC International Fellowship.

The second and third named authors were partially supported by NSERC grants 141066-04 and OGP0005616 respectively. 
continua. However, the situation for maps of graphs with hereditarily indecomposable limits is slightly different. Block, Keesling and Uspenskij [4] have shown that the continuous surjections $g$ of $[0,1]$ such that $([0,1], g)$ is hereditarily indecomposable form a nowhere dense set in the space of continuous surjections of $[0,1]$. In [6] and [10], two very different surjections of $[0,1]$ onto itself are constructed, both of which yield the pseudo-arc (the topologically unique hereditarily indecomposable arc-like continuum) as their inverse limits.

The map $f:[0,1] \rightarrow[0,1]$ constructed by Henderson in [6] is simple from the point of view of topological dynamics. It has exactly two fixed points 0 and 1 , and every point $x$ different from 1 satisfies $\lim _{n \rightarrow \infty} f^{n}(x)=0$. In contrast, the map $f:[0,1] \rightarrow[0,1]$ constructed by Minc and Transue in [10] is topologically transitive, that is, there exists a point $x$ such that the subset $\left\{f^{n}(x) \mid n \geq 0\right\}$ is dense in $[0,1]$. Thus it seems to be difficult to obtain a characterization of the dynamics of maps which yield hereditarily indecomposable inverse limits (see also Kuykendall [8]). The purpose of the present paper is to prove the following theorem, generalizing the construction of the Minc-Transue map above.

Main Theorem. Let $G$ be a compact connected graph and $f: G \rightarrow G$ a piecewise linear surjection which satisfies the following condition:

(*) for each nondegenerate subcontinuum $A$ of $G$, there is a positive integer $n$ such that $f^{n}(A)=G$.

Then for each $\varepsilon>0$, there is a map $f_{\varepsilon}: G \rightarrow G$ which is $\varepsilon$-close to $f$ such that $\left(G, f_{\varepsilon}\right)$ is hereditarily indecomposable.

The proof of the Main Theorem follows that in [10], where $G$ is assumed to be an arc. We construct inductively small "crooked perturbations" of a given map to obtain the desired map as the limit of a sequence of these perturbations. Our main result extends the Minc-Transue theorem [10] to maps of compact connected graphs satisfying condition $(*)$. Another perturbation of the Minc-Transue map has been considered in [9] in a different context. Also a similar set of conditions has been considered by Williams in [12] and [13]. His local expansions on branched 1-manifolds satisfying Axioms 0-3 ([12], [13]) satisfy the conditions of our main theorem.

2. Preliminaries. In this section, we recall some definitions and results which are necessary for the proof of the main theorem.

Definition 2.1. (1) A metric $\varrho$ on a graph $G$ is called a convex (or arc length) metric if, for each pair of points $x$ and $y$ of $G$, there is an isometry $\alpha$ of the interval $[0, \varrho(x, y)]$ in the real line into $G$ such that $\alpha(0)=x$ and $\alpha(\varrho(x, y))=y$. 
(2) Let $G$ be a graph with a convex metric $\varrho$. For a subset $A$ of $G$, $\operatorname{diam}(A)$ denotes the diameter of $A$ with respect to $\varrho$. The $\varepsilon$-neighborhood of $A$ (with respect to $\varrho$ ) is denoted $N_{\varepsilon}(A)$. The interior and closure of $A$ are denoted by $\operatorname{int}(A)$ and $\operatorname{cl}(A)$, respectively.

Definition 2.2. Let $f: G \rightarrow G$ be a map of a graph $G$ with metric $\varrho$ and let $\varepsilon>0$. A map $\omega:[0,1] \rightarrow G$ is said to be $(f, \varepsilon)$-crooked if there exist $s$ and $t$ with $0<s \leq t<1$ such that $\varrho(f \circ \omega(s), f \circ \omega(1)) \leq \varepsilon$ and $\varrho(f \circ \omega(t), f \circ \omega(0)) \leq \varepsilon$.

Remark. In Definition 2.2, notice that, if there exist intervals $I_{1}$ and $I_{2}$ in $[0,1]$ disjoint except possibly for a common endpoint and such that $f(\omega([0,1])) \subset \operatorname{cl}\left(N_{\varepsilon}\left(f\left(\omega\left(I_{i}\right)\right)\right)\right)$ for $i=1,2$, then $\omega$ is $(f, \varepsilon)$-crooked.

First, we state three propositions without proof. They are analogues of Propositions $1-3$ of $[10]$ and the proofs are simple.

Proposition 2.3. Let $f: G \rightarrow G$ be a map of a graph $G$. If $\alpha \geq \delta$, then every $\omega:[0,1] \rightarrow G$ which is $(f, \delta)$-crooked is also $(f, \alpha)$-crooked.

Proposition 2.4. Let $G$ be a graph with metric $\varrho$ and let $f, g: G \rightarrow G$ be two maps on $G$.

(1) Suppose $\varrho(f(t), g(t))<\varepsilon$ for each $t \in G$. If a map $\omega:[0,1] \rightarrow G$ is $(f, \delta)$-crooked, then $\omega$ is also $(g, \delta+2 \varepsilon)$-crooked.

(2) Suppose that, for each pair of points $x, y \in G$ with $\varrho(x, y)<\delta$, we have $\varrho(g(x), g(y))<\varepsilon$. Then a map $\omega:[0,1] \rightarrow G$ which is $(f, \delta)$-crooked is also $(g \circ f, \varepsilon)$-crooked.

Proposition 2.5. Let $G$ be a graph and $\delta>0$. Let $\left\{f_{i} \mid i=1,2, \ldots\right\}$ be a sequence of maps of $G$ onto itself which converges uniformly to $f$. If a map $\omega:[0,1] \rightarrow G$ is $\left(f_{i}, \delta\right)$-crooked for each $i=1,2, \ldots$, then $\omega$ is $(f, \delta)$-crooked.

The proof of the following proposition is similar to that of Proposition 4 of [10]. For the sake of completeness we provide the proof.

Proposition 2.6. Let $f: G \rightarrow G$ be a map such that for each $\delta>0$, there is an integer $n>0$ such that each map $\omega:[0,1] \rightarrow G$ is $\left(f^{n}, \delta\right)$-crooked. Then the inverse limit $(G, f)$ is hereditarily indecomposable.

Proof. Let $X$ denote the inverse limit $(G, f)$. Suppose that $A$ and $B$ are subcontinua of $X$ such that $A \cap B \neq \emptyset, B \backslash A \neq \emptyset$ and $A \backslash B \neq \emptyset$. Let $k$ be an integer so large that, if $\pi_{k}: X \rightarrow G$ is the $k$ th-coordinate projection, then $\pi_{k}(A) \backslash \pi_{k}(B) \neq \emptyset$ and $\pi_{k}(B) \backslash \pi_{k}(A) \neq \emptyset$. Let $\delta>0$ be such that there exists $x \in \pi_{k}(A) \backslash N_{\delta}\left(\pi_{k}(B)\right)$ and $y \in \pi_{k}(B) \backslash N_{\delta}\left(\pi_{k}(A)\right)$. Let $n$ be an integer such that each map $\omega:[0,1] \rightarrow G$ is $\left(f^{n}, \delta\right)$-crooked. Let $\omega:[0,1] \rightarrow \pi_{n+k}(A \cup B)$ 
be an embedding so that $f^{n}(\omega(0))=x, f^{n}(\omega(1))=y$ and $\pi_{n+k}(A) \cap \omega([0,1])$ is a connected set. Then $\omega$ is not $\left(f^{n}, \delta\right)$-crooked. This is a contradiction.

The following proposition is an analogue of Proposition 6 of [10].

Proposition 2.7. Let $f: G \rightarrow G$ be a map which satisfies condition (*). Then for each $\varepsilon>0$, there exists an integer $n>0$ such that for each subcontinuum $A$ with $\operatorname{diam}(A) \geq \varepsilon, f^{n}(A)=G$.

Proof. Choose pairwise disjoint $\operatorname{arcs} K_{1}, \ldots, K_{n}$ in $G$ with $\operatorname{diam}\left(K_{i}\right) \leq$ $\varepsilon / 4$ for each $i=1, \ldots, n$ such that each continuum in $G \backslash\left(K_{1} \cup \cdots \cup K_{n}\right)$ has diameter $<\varepsilon / 2$. Let $m_{i}$ be a positive integer such that $f^{m_{i}}\left(K_{i}\right)=G$. Let $m=\max \left\{m_{1}, \ldots, m_{n}\right\}$. If $A$ is a continuum in $G$ with $\operatorname{diam}(A) \geq$ $\varepsilon$, then $A$ contains $K_{i}$ for some $i=1, \ldots, n$. Hence $f^{m}(A) \supset f^{m}\left(K_{i}\right)=$ $f^{m-m_{i}}\left(f^{m_{i}}\left(K_{i}\right)\right)=f^{m-m_{i}}(G)=G$.

3. Proof of Main Theorem. Our proof of the Main Theorem follows that in [10]. We prove that each piecewise linear map of a graph which satisfies $(*)$ can be perturbed slightly so as to have a local expansion property (Lemma 3.2) and further to have a certain crookedness (Lemma 3.3). The second perturbation corresponds to Lemma in [10] (pp. 1167-1168) and the first perturbation provides a situation to which the second perturbation can be applied. Once we obtain these results, the remaining part of the proof proceeds as in [10]. First we prove the following lemma which is an analogue of Proposition 5 of [10].

By a $f a n$, we mean a wedge of finitely many arcs. A map $f: X \rightarrow Y$ of a compact, metric space $X$ to a continuum $Y$ is said to be weakly confluent if for each subcontinuum $K \subset Y$, there exists a subcontinuum $L \subset f^{-1}(K)$ such that $f(L)=K$. It is well known that every map of a continuum onto an arc is weakly confluent.

Convention. Throughout this section, we fix a convex metric $\varrho$ and a triangulation $\mathcal{T}$ on a compact connected graph $G$ such that each edge $e$ of $\mathcal{T}$ is isometric to the unit interval. Let $\operatorname{Id}_{H}$ denote the identity map on a subgraph $H$.

In what follows, we often consider a map $g_{R}: R \rightarrow R^{*}$ defined on an edge $R$ of a triangulation of $G$ to a subgraph $R^{*} \subset G$. We apply Definition 2.2 to $g_{R}$ with the domain $R$ of $g_{R}$ being regarded as the unit interval to say simply that " $g_{R}$ is $\left(\operatorname{Id}_{R^{*}}, \gamma / 5\right)$-crooked" etc.

Note that the numbers $\gamma / 5$ and $\varepsilon / 4 k$ in the statement of Lemma 3.1 are chosen for a technical reason for later use.

LEMMA 3.1. Let $G$ be the above compact connected graph with a triangulation $\mathcal{T}$. For each well-chosen $k, \varepsilon$ and $\gamma$ with $k \geq 1$ an integer, 
$0<\gamma<\varepsilon / 4 k$ and $0<\varepsilon<1 / 4$, there exist a piecewise linear map $g: G \rightarrow G$ and a subdivision $\mathcal{T}^{\prime}$ of $\mathcal{T}$ which satisfy the following conditions:

(1) Each edge of $\mathcal{T}^{\prime}$ has length $\gamma / 5$.

(2) For each edge $R$ of $\mathcal{T}^{\prime}$, we have $g(R)=R^{*}$, where $R^{*}$ is the largest subpolyhedron of $\mathcal{T}^{\prime}$ contained in the closure of the $\varepsilon / 2$-neighborhood of $R$. In particular, $\operatorname{diam}(g(R)) \geq \varepsilon / 2$.

(3) For each edge e of $\mathcal{T}$, there exists an edge $R$ of $\mathcal{T}^{\prime}$ such that each edge of $\mathcal{T}^{\prime}$ which meets $R^{*}$ is contained in e.

(4) For each subcontinuum $A$ of $G$ with $\operatorname{diam}(A) \geq \gamma / 5$ and for each positive number q, we have

$$
A \subset g(A) \subset g\left(N_{q}(A)\right) \subset N_{q+\gamma / 5}(g(A)) .
$$

(5) For each subcontinuum $B$ of $G$ with $\operatorname{diam}(B) \leq \gamma / 5$,

$$
\operatorname{diam}(g(B)) \geq 2 k \operatorname{diam}(B) \geq 2 \operatorname{diam}(B) .
$$

(6) Each map $\omega:[0,1] \rightarrow G$ with $\operatorname{diam}(\omega([0,1]))<\gamma / 5$ is $(g, 2 \gamma / 5)$ crooked.

(7) $\varrho\left(g, \operatorname{Id}_{G}\right)<\varepsilon / 2+\gamma / 5$.

REMARK. The following fact, an easy consequence of (1) and the definition of $R^{*}$, is important in the proof below: If $R_{1}$ and $R_{2}$ are adjacent edges of $\mathcal{T}^{\prime}$, then the Hausdorff distance of $R_{1}^{*}$ and $R_{2}^{*}$ is at most $\gamma / 5$.

Proof of Lemma 3.1. Let $G, \mathcal{T}, k, \varepsilon$ and $\gamma$ be as in the hypothesis. By replacing $\gamma$ by a smaller number if necessary, we may suppose that $1 / \gamma$ is an integer. Let $\mathcal{T}^{\prime}$ be a subdivision of $G$ satisfying (1) and (3). Notice that for each edge $R$ of $\mathcal{T}^{\prime}, R^{*}$ is a fan containing at most one vertex of $\mathcal{T}$. Denote the set of all edges of $\mathcal{T}$ by $\left\{e_{1}, \ldots, e_{m}\right\}$. For each $j=1, \ldots, m$, take an edge $R_{j}$ of $\mathcal{T}^{\prime}$ satisfying (3). Then

$$
\operatorname{cl}\left(G-\bigcup_{j=1}^{m} R_{j}\right)=\bigcup_{i=1}^{s} T_{i},
$$

where the $T_{i}$ 's are pairwise disjoint fans such that $T_{i}$ contains exactly one vertex $v_{i}$ of $\mathcal{T}$. Label the edges of $\mathcal{T}$ which meet $v_{i}$ as $e_{i, 1}, \ldots, e_{i, k_{i}}$.

Let $R$ be an edge of $\mathcal{T}^{\prime}$ other than $R_{1}, \ldots, R_{m}$. Then $R \subset T_{i}$ for some $i$ and hence $R^{*} \subset \bigcup_{j=1}^{k_{i}} e_{i_{j}}$ by the definition of $R^{*}$. Let $v_{R, 0}$ and $v_{R, 1}$ be the endpoints of $R$, where $v_{R, 0}$ is the endpoint of $R$ closer to $v_{i}$.

Next we define a piecewise linear map $\phi_{R}:[0,1] \rightarrow R^{*}$ as follows. If $R^{*} \subset e_{i, j}$ for some $j$, then let $a_{t}$ be the endpoint of $R^{*}$ closer to $v_{R, t}$ for $t=0,1$. The map $\phi_{R}:[0,1] \rightarrow R^{*}$ is the simplest piecewise linear map which carries the 5 -tuple $(0,1 / 4,1 / 2,3 / 4,1)$ onto $\left(v_{R, 0}, a_{1}, a_{0}, a_{1}, v_{R, 1}\right)$ (see Figure 1). 


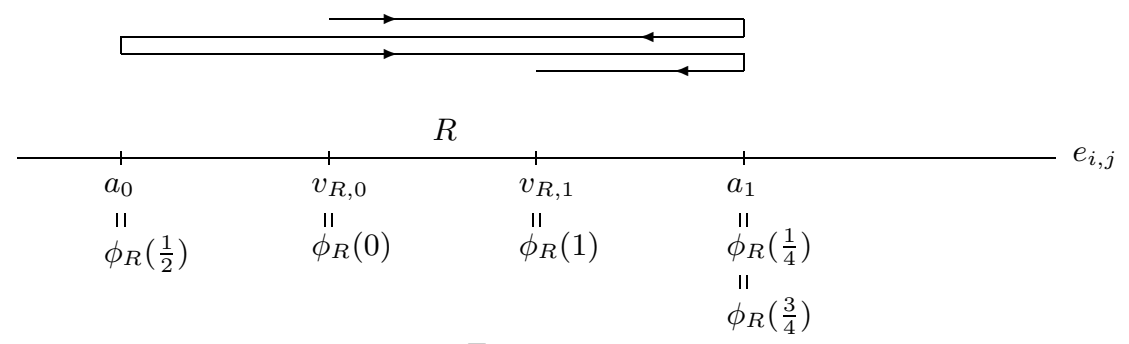

Fig. 1

Now suppose $R^{*}$ is not a subset of $e_{i, j}$ for any $j$. The subset $R^{*}$ is a fan with the unique branch point $v_{i}$. Let $a_{j}$ be the endpoint of $R^{*}$ in $e_{i, j}$ for $j=$ $1, \ldots, k_{i}$ (note that $a_{j}$ is distinct from $v_{i}$ ). Let $\xi=2 k_{i}$ and let $\phi_{R}:[0,1] \rightarrow R^{*}$ be the simplest piecewise linear map which carries the $(\xi+1)$-tuple $(0,1 / \xi, 2 / \xi, \ldots,(\xi-1) / \xi, 1)$ onto $\left(v_{R, 0}, a_{1}, a_{2}, \ldots, a_{k_{i}}, a_{k_{i}-1}, \ldots, a_{1}, v_{R, 1}\right)$ (see Figure 2).

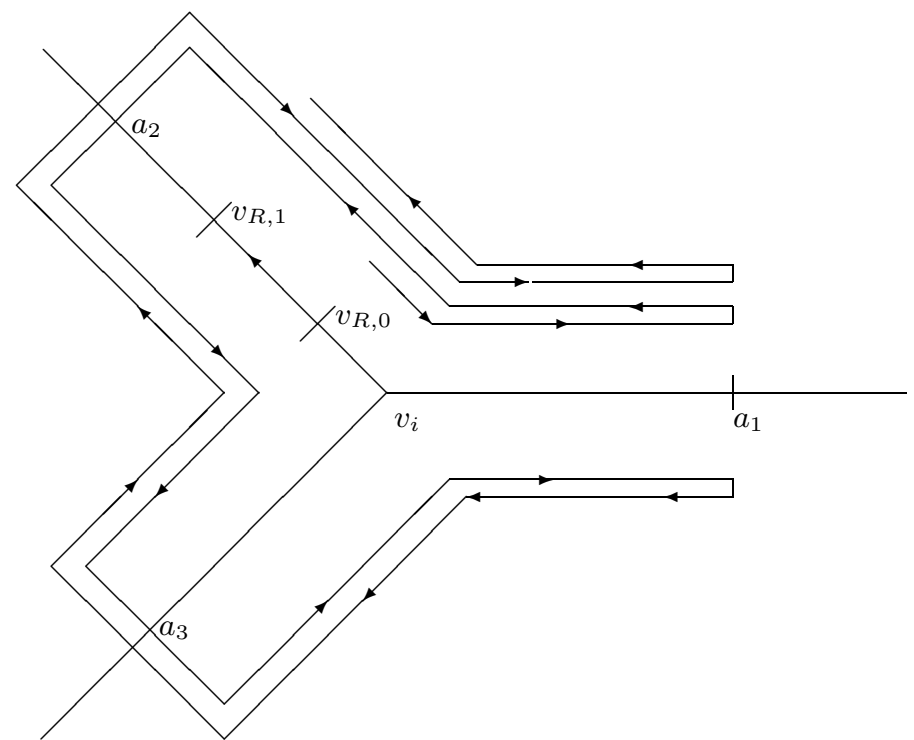

Fig. 2

Next we define an $\left(\operatorname{Id}_{[0,1]}, p\right)$-crooked piecewise linear map $b_{R}: R \rightarrow[0,1]$ with $b_{R}\left(v_{R, 0}\right)=0$ and $b_{R}\left(v_{R, 1}\right)=1$, where the positive number $p$ is chosen so that

$$
\varrho\left(z_{1}, z_{2}\right)<p \Rightarrow \varrho\left(\phi_{R}\left(z_{1}\right), \phi_{R}\left(z_{2}\right)\right)<\gamma / 5,
$$

where $z_{1}, z_{2} \in R$. Hence by Proposition 2.4(2), the map $g_{R}=\phi_{R} \circ b_{R}: R \rightarrow$ $R^{*}$ is $\left(\operatorname{Id}_{R^{*}}, \gamma / 5\right)$-crooked.

We define $b_{R}: R \rightarrow[0,1]$ to be a nowhere locally constant simplicial map with respect to some subdivisions $\mathcal{P}$ and $\mathcal{Q}$ of $R$ and $[0,1]$ respectively. Let 
$v_{R, 0}=p_{0}, p_{1}, \ldots, p_{M-1}, p_{M}=v_{R, 1}$ be the vertices of $\mathcal{P}$ so that $p_{i}$ and $p_{i+1}$ are adjacent. We also assume that the midpoint of $R$ is a vertex $p_{\mu}$ of $\mathcal{P}$. Similarly let $0=q_{0}<q_{1}<\cdots<q_{N}=1$ be the vertices of $\mathcal{Q}$. The map $b_{R}$ satisfies the following conditions:

(crookedness in the sense of Bing [3]) If $b_{R}\left(p_{s}\right)=q_{u}$ and $b_{R}\left(p_{t}\right)=q_{v}$ with $v-u \geq 5$, then there exist $x$ and $y$ with $s<x<y<t$ such that $b_{R}\left(p_{x}\right)=q_{v-1}$ and $b_{R}\left(p_{y}\right)=q_{u+1}$, and

$b_{R}\left(\left[v_{R, 0}, p_{\mu}\right]\right) \supset[0,1 / 2]$ and $b_{R}\left(\left[p_{\mu}, v_{R, 1}\right]\right) \supset[1 / 2,1]$, where $\left[v_{R, 0}, p_{\mu}\right]$ is the segment in $R$ connecting $v_{R, 0}$ with $p_{\mu}$, etc.

We further choose $\mathcal{P}$ and $\mathcal{Q}$ so that $\varrho\left(p_{i}, p_{i+1}\right)=\gamma / 5 M$ for $i=1, \ldots, M-1$ and $q_{j+1}-q_{j}=1 / N$ for $j=1, \ldots, N-1$. By the crookedness condition (9), $M / N$ becomes arbitrarily large as $M$ increases to infinity. So the derivative of $b_{R}$ becomes arbitrarily large in absolute value for large $N$.

As we stated above, we define $g_{R}=\phi_{R} \circ b_{R}: R \rightarrow R^{*}$ for each edge $R$ other than $R_{1}, \ldots, R_{m}$. Recall from the construction that $\phi_{R}$ "contracts" subintervals of $[0,1]$ by a factor of order at most $1 / \varepsilon$. Therefore, by taking $N$ large enough, we obtain the following condition:

$$
\operatorname{diam}\left(g_{R}(B)\right) \geq 4 k \operatorname{diam}(B) \quad \text { for each subinterval } B \text { in } R \text {. }
$$

Also we may require

$g_{R}$ is $\left(\operatorname{Id}_{R^{*}}, \gamma / 5\right)$-crooked on each interval $J$ of $R$.

Indeed, if $b_{R}(J)$ contains five consecutive edges of $\mathcal{Q}$, then (9) guarantees the desired crookedness. Otherwise, $\operatorname{diam}\left(b_{R}(J)\right)<6 / N$ and hence $\operatorname{diam}(g(J))<\gamma / 5$, by taking sufficiently large $N$. It follows that $g_{R} \mid J$ is trivially $\left(\operatorname{Id}_{R^{*}}, \gamma / 5\right)$-crooked.

By the construction of $\phi_{R}$, we have

$$
\begin{aligned}
& g_{R}(B)=R^{*} \text { for each subinterval } B \text { containing an endpoint of } R \text { and } \\
& \text { having length at least } \gamma / 10 \text {. }
\end{aligned}
$$

We next define $g_{R_{i}}: R_{i} \rightarrow R_{i}^{*}$ for $i \in\{1, \ldots, m\}$. Let $P$ and $Q$ be the edges of $\mathcal{T}^{\prime}$ adjacent to $R_{i}$. Note that $P^{*} \cup R_{i}^{*} \cup Q^{*} \subset e_{i}$ by (3). We suppose $P \subset T_{k}$ and $Q \subset T_{j}$ for some $k \neq j$.

The vertex $v_{P, 0}$ is closer to $v_{k}$ than $v_{P, 1}$ is, and $v_{Q, 0}$ is closer to $v_{j}$ than $v_{Q, 1}$ is. So $v_{P, 1}$ is the common endpoint of $P$ and $R_{i}$, and $v_{Q, 1}$ is the common endpoint of $Q$ and $R_{i}$. Let $a_{k}$ be the endpoint of $R_{i}^{*}$ closer to $v_{k}$, and $a_{j}$ the endpoint of $R_{i}^{*}$ closer to $v_{j}$. Let $\phi_{R_{i}}:[0,1] \rightarrow R_{i}^{*}$ be the simplest piecewise linear map which carries $(0,1 / 5,2 / 5,3 / 5,4 / 5,1)$ onto $\left(v_{P, 1}, a_{j}, a_{k}, a_{j}, a_{k}, v_{Q, 1}\right)$. Let $b_{R_{i}}: R_{i} \rightarrow[0,1]$ be an $\left(\operatorname{Id}_{[0,1]}, p\right)$-crooked piecewise linear map with $b_{R_{i}}\left(v_{P, 1}\right)=0$ and $b_{R_{i}}\left(v_{Q, 1}\right)=1$ for $p$ as in (8). With the same conditions as used in defining $b_{R}$ above, let $g_{R_{i}}=\phi_{R_{i}} \circ b_{R_{i}}$. Then 


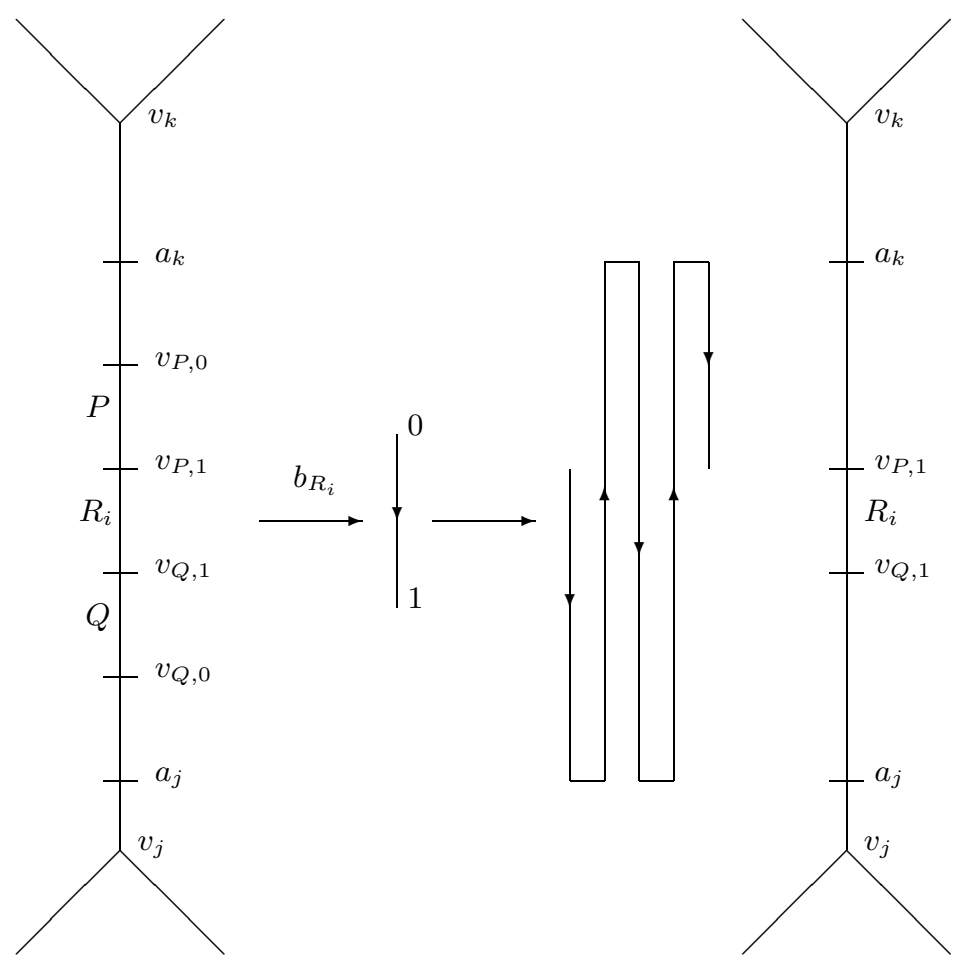

Fig. 3

(14) $g_{R_{i}}$ is $\left(\operatorname{Id}_{R_{i}^{*}}, \gamma / 5\right)$-crooked on each subinterval of $R_{i}$, and

(15) $\operatorname{diam}\left(g_{R_{i}}(B)\right) \geq 4 k \operatorname{diam}(B)$ for each subinterval $B$ of $R_{i}$ (see Figure 3$)$.

Let $g: G \rightarrow G$ be a map defined by $g=\bigcup\left\{g_{R}: R \in \mathcal{R}_{\mathcal{T}^{\prime}}\right\}$. Then $g$ is well defined and continuous since the endpoints of $R$ are fixed by $g_{R}$ for each edge $R$ of $\mathcal{T}^{\prime}$.

Condition (5) follows from (11) and (15) above. Also (7) follows from the fact that $g(R)=R^{*}$ and by definition of $R^{*}$ for an edge $R$ of $\mathcal{T}^{\prime}$.

Now we are going to prove (4). Let $A$ be a subcontinuum of $G$ with $\operatorname{diam}(A) \geq \gamma / 5$ and let $q>0$. As each edge of $\mathcal{T}$ has length $\gamma / 5$, there exist $R$ in $\mathcal{T}^{\prime}$ and $v$ an endpoint of $R$ such that the component $B$ of $v$ in $R \cap A$ has diameter at least $\gamma / 10$. Hence by $(10), R^{*} \subset g(A)$. Let $q=$ $\alpha \gamma / 5+\beta$, where $\alpha$ is a nonnegative integer and $0 \leq \beta \leq \gamma / 5$, and take a point $x \in A$ with $\varrho(x, A \cap R)=q$. Since $g$ maps adjacent edges of $\mathcal{T}^{\prime}$ to within Hausdorff distance $\gamma / 5$ of each other (see the Remark before the proof), we have $\varrho\left(g(x), R^{*}\right) \leq \alpha \gamma / 5+\beta+\gamma / 5 \leq q+\gamma / 5$. Thus $g\left(N_{q}(A)\right) \subset$ $N_{q+\gamma / 5}(g(A))$. The other inclusions follow easily from the construction of $g$. This proves (4). 
It remains to show that (6) is satisfied. Let $\omega:[0,1] \rightarrow G$ be a map with $\operatorname{diam}(\omega([0,1]))<\gamma / 5$. If $\operatorname{diam} g(\omega([0,1])) \leq 2 \gamma / 5$, then $\omega$ is trivially $(g, 2 \gamma / 5)$-crooked. If diam $g(\omega([0,1]))>2 \gamma / 5$, we have two cases to consider.

CASE A: $\omega([0,1]) \subset R$ for some edge $R$ of $\mathcal{T}^{\prime}$. As $\left.g\right|_{\omega([0,1])}=\left.g_{R}\right|_{\omega([0,1])}$ is $\left(\operatorname{Id}_{R^{*}}, \gamma / 5\right)$-crooked by (12) and (14), there exist intervals $K_{1}$ and $K_{2}$ in $\omega([0,1])$ disjoint except possibly for a common endpoint such that

$$
g_{R}(\omega([0,1])) \subset \operatorname{cl}\left(N_{\gamma / 5}\left(g_{R}\left(K_{j}\right)\right)\right), \quad j=1,2 .
$$

Using weak confluence of $\omega$, we find intervals $I_{1}$ and $I_{2}$ in $[0,1]$ disjoint except possibly for a common endpoint such that $\omega\left(I_{j}\right)=K_{j}$. This proves that $\omega$ is $(g, \gamma / 5)$-crooked in this case.

CASE B: $\omega([0,1])$ is not contained in any edge of $\mathcal{T}^{\prime}$. As $\operatorname{diam}(\omega([0,1]))$ $<\gamma / 5$, there is a vertex $v$ of $\mathcal{T}^{\prime}$ such that $v \in \omega([0,1]) \subset \operatorname{int}\left(\operatorname{st}\left(v, \mathcal{T}^{\prime}\right)\right)$, where $\operatorname{st}\left(v, \mathcal{T}^{\prime}\right)$ is the union of the edges of $\mathcal{T}^{\prime}$ having $v$ as an endpoint. By construction of $g$, it follows that there is $R$ with $v$ being an endpoint such that $\operatorname{cl}\left(N_{\gamma / 5}(g(R \cap \omega([0,1])))\right) \supset g(\omega([0,1]))$. Since $R \cap \omega([0,1])$ is a subinterval of $R$ and $g$ is $\left(\operatorname{Id}_{R^{*}}, \gamma / 5\right)$-crooked on all subintervals of $R$, by (11) and (12), there exist intervals $K$ and $L$, disjoint except possibly for a common endpoint, such that

$$
g(R \cap \omega([0,1])) \subset \operatorname{cl}\left(N_{\gamma / 5}(K)\right) \cap \operatorname{cl}\left(N_{\gamma / 5}(L)\right) .
$$

Hence $g(\omega([0,1])) \subset \operatorname{cl}\left(N_{2 \gamma / 5}(K)\right) \cap \operatorname{cl}\left(N_{2 \gamma / 5}(L)\right)$ and in this case also, $\omega$ is $(g, 2 \gamma / 5)$-crooked and (6) is proved.

This completes the proof of Lemma 3.1.

REMARK. It follows from the construction of $g$ that, for each edge $R$ of $\mathcal{T}^{\prime}$, there exist $\operatorname{arcs} K_{0}$ and $K_{1}$, disjoint except possibly for a common endpoint, in $R$ such that $g(R)=g\left(K_{j}\right)$ for $j=0,1$.

The next lemma describes a perturbation of $f$ of the main theorem to a map $f_{0}$ with local expansion property, while retaining condition $(*)$.

Lemma 3.2. Let $f: G \rightarrow G$ be a piecewise linear map which satisfies $(*)$. Then, for each $\delta$ with $0<\delta<1$, there exist a piecewise linear map $f_{0}$ : $G \rightarrow G$ and a positive number $\gamma<\delta$ such that

(1) $\varrho\left(f_{0}, f\right)<\delta$.

(2) For each subcontinuum $A$ in $G$ with $\operatorname{diam}(A)<\gamma / 5$,

$$
\operatorname{diam}\left(f_{0}(A)\right) \geq 2 \operatorname{diam}(A) .
$$

(3) The map $f_{0}$ satisfies condition $(*)$.

REMARK. 1) As is seen below, $f_{0}=f \circ g$, where $g$ is the map obtained in Lemma 3.1. The crookedness condition (9) of Lemma 3.1 plays no role in the proof of this lemma. We will make use of (9) later in Lemma 3.3. 
2) The proof below shows that the map $f_{0}$ satisfies the following condition: there exists an integer $n>0$ such that, for every subcontinuum $A$ of $G$ such that $\operatorname{diam}(A) \geq \gamma / 5$, we have $f_{0}^{n}(A)=G$.

Proof of Lemma 3.2. Observe first that $\operatorname{diam}(G) \geq 1$. As $f$ is piecewise linear and nowhere locally constant, there exist $0<S_{1}<1<S_{2}$ such that $S_{1}^{-1}$ and $S_{2}$ are integers and

$$
S_{1} \operatorname{diam}(C) \leq \operatorname{diam}(f(C)) \leq S_{2} \operatorname{diam}(C)
$$

for each subcontinuum $C$ of $G$.

Let $k=2 / S_{1}$ and let $\varepsilon>0$ be a number such that

$$
\varepsilon<\delta / 2 S_{2} \text {. }
$$

By Proposition 2.7, there exists a positive integer $n$ such that

$$
f^{n}(A)=G \quad \text { for each subcontinuum } A \text { of } G \text { with } \operatorname{diam}(A)>\varepsilon / 4 .
$$

Take a positive number $\gamma$ so that $1 / \gamma$ is an integer and

$$
\gamma<\min \left\{\varepsilon / 2,1 / S_{2}^{n}\right\}
$$

Apply Lemma 3.1 to the above $k, \varepsilon$ and $\gamma$, to obtain a map $g: G \rightarrow G$ which is $(\varepsilon / 2+\gamma / 5)$-close to $\operatorname{Id}_{G}$ and a triangulation $\mathcal{T}^{\prime}$ of $G$ which satisfy conditions (1)-(7) of Lemma 3.1. Define a map $f_{0}: G \rightarrow G$ by $f_{0}=f \circ g$. We prove that $f_{0}$ satisfies the desired conditions.

Condition (1) follows immediately from (4)-(6) above and the condition (7) of Lemma 3.1.

Let $A$ be a subcontinuum of $G$ with $\operatorname{diam}(A) \geq \gamma / 5$. As each edge of $\mathcal{T}^{\prime}$ has length equal to $\gamma / 5$, there exists an edge $R$ of $\mathcal{T}^{\prime}$ such that $\operatorname{diam}(A \cap R) \geq \gamma / 10$. By (10) of Lemma 3.1, we have $R^{*} \subset g(A)$. Also by (2) of Lemma 3.1, we see that $\operatorname{diam}(g(A)) \geq \varepsilon / 2$. By the choice of $n$, we have $f^{n}(g(A))=G$. From $\gamma<1 / S_{2}^{n}$ by (7), it follows from (5) and $f^{n}(g(A))=G$ that $\operatorname{diam}\left(f^{j}(g(A))\right) \geq \gamma$ for each $j \geq 1(\operatorname{recall} \operatorname{diam}(G) \geq 1)$. By (4) of Lemma 3.1, $g\left(f^{j}(g(A))\right) \supset f^{j}(g(A))$ for each $j \geq 1$. Therefore $f_{0}(f(g(A)))=f g f(g(A)) \supset f^{2}(g(A))$, and an inductive argument shows that $f_{0}^{j-1}(f(g(A))) \supset f^{j}(g(A))$ for each $j \geq 1$. Thus, $f_{0}^{n}(A)=$ $f_{0}^{n-1}(f(g(A))) \supset f^{n}(g(A))=G$, as required.

To prove (2), let $A$ be a nondegenerate subcontinuum of $G$. If $\operatorname{diam}(A)$ $\geq \gamma / 5$, then $f_{0}^{n}(A)=G$ by the above. Suppose, therefore, that $\operatorname{diam}(A)$ $<\gamma / 5$. We prove first that $\operatorname{diam}\left(f_{0}(A)\right) \geq 2 \operatorname{diam}(A)$. By (5) of Lemma 3.1, $\operatorname{diam}(g(A)) \geq 2 k \operatorname{diam}(A)>\left(2 / S_{1}\right) \operatorname{diam}(A) \geq 4 \operatorname{diam}(A)$. So

$$
\begin{aligned}
\operatorname{diam}\left(f_{0}(A)\right) & =\operatorname{diam}(f(g(A))) \geq S_{1} \operatorname{diam}(g(A)) \\
& \geq S_{1} \frac{2}{S_{1}} \operatorname{diam}(A)=2 \operatorname{diam}(A),
\end{aligned}
$$


which proves (2). If $\operatorname{diam}\left(f_{0}(A)\right) \geq \gamma / 5$, then $f_{0}^{n+1}(A)=G$, and if $\operatorname{diam}\left(f_{0}(A)\right)<\gamma / 5$, then $\operatorname{diam}\left(f_{0}^{2}(A)\right) \geq 2^{2} \operatorname{diam}(A)$. By repeating this argument finitely many times we see that $f_{0}^{m}(A)=G$ for some integer $m$. So (3) is satisfied.

This completes the proof.

The map $f_{0}$ of Lemma 3.2 is further perturbed to obtain, by induction, a sequence of maps so that the limit map has the crookedness property required in the hypothesis of Proposition 2.6. The following lemma describes the general inductive step.

Lemma 3.3. Let $h: G \rightarrow G$ be a piecewise linear map which satisfies (*) and the condition: there exists $0<\beta<1$ such that

$(+)_{\beta}$ for each subcontinuum $A$ with $\operatorname{diam}(A) \leq \beta$,

$$
\operatorname{diam}(h(A)) \geq 2 \operatorname{diam}(A) .
$$

Then, for each $\delta$ with $0<\delta<1$, there exist a map $F: G \rightarrow G$ satisfying $(*)$, a positive number $\gamma<\delta$ and an integer $n>0$ such that

(1) $\varrho(F, h)<\delta$.

(2) $\quad F^{n}(A)=G$ for every subcontinuum $A$ of $G$ with $\operatorname{diam}(A) \geq \gamma / 5$.

(3) The map $F$ satisfies condition $(+)_{\gamma / 5}$.

(4) Each map $\omega:[0,1] \rightarrow G$ is $\left(F^{n}, \delta\right)$-crooked.

Proof. As in Lemma 3.2, choose $S_{1}$ and $S_{2}$ so that $S_{1}^{-1}$ and $S_{2}$ are integers, $0<S_{1}<1<S_{2}$ and

$$
\begin{aligned}
S_{1} \operatorname{diam}(C) \leq \operatorname{diam}(h(C)) \leq S_{2} \operatorname{diam}(C) & \text { for each subcontinuum } C \text { of } G .
\end{aligned}
$$

Let $k=2 / S_{1}$. Next choose $\varepsilon$ so that each subcontinuum of $G$ with diameter less than $\varepsilon$ contains at most one branch point of $G$ and

$$
\varepsilon<\frac{\delta}{2 S_{2}}
$$

and an integer $n$ (using Proposition 2.7) in such a way that

$$
h^{n}(A)=G \text { for each subcontinuum } A \text { of } G \text { with } \operatorname{diam}(A)>\varepsilon / 4 .
$$

The choice of a positive number $\gamma$ is more delicate than in Lemma 3.2. The $\gamma$ is chosen as follows:

$$
\gamma<\min \left\{\frac{\left(S_{2}-1\right) \varepsilon}{8 S_{2}^{n+1}}, \frac{\varepsilon}{4 k}, \frac{S_{1}}{4 S_{2}^{n}}, \frac{\beta}{4 S_{2}^{n}}\right\} .
$$

We again apply Lemma 3.1 to obtain a map $g: G \rightarrow G$ and a triangulation $\mathcal{T}^{\prime}$ of $G$ which satisfy conditions (1)-(7) of Lemma 3.1. Define a map $F: G \rightarrow G$ by $F=h \circ g$. We prove that $F$ satisfies the desired conditions. 
As $h$ satisfies the hypothesis of Lemma 3.2, conditions (1)-(3) follow from Lemma 3.2. We need only show that (4) holds for $F$.

We will need the following estimation. Since $h$ satisfies $(+)_{\beta}$ and $\gamma<\beta / 2$ (see (8)), $h$ satisfies

(9) $\operatorname{diam}(h(A)) \geq 2 \min \{\operatorname{diam}(A), \gamma / 5\} \quad$ for each subcontinuum $A$.

We also need the following inclusion:

(10) For each subcontinuum $A$ of $G$ with $\operatorname{diam}(A) \geq \gamma / 5$, for each positive number $q$, and for each positive integer $j$, we have

$$
F^{j}\left(N_{q}(A)\right) \subset N_{S_{2}^{j}(q+2 \gamma)}\left(F^{j}(A)\right) .
$$

Indeed, for each subcontinuum $A$ of $G$ with $\operatorname{diam}(A) \geq \gamma / 5$ and each $q>0$,

$$
\begin{aligned}
F\left(N_{q}(A)\right)=h\left(g\left(N_{q}(A)\right)\right) & \subset h\left(N_{q+\gamma / 5}(g(A))\right) \\
& \subset N_{S_{2}(q+\gamma / 5)}(h(g(A)))=N_{S_{2}(q+\gamma / 5)}(F(A))
\end{aligned}
$$

by (5) and Lemma 3.1(4). Inductively, using (5) and Lemma 3.1(4),

$$
F^{j}\left(N_{q}(A)\right) \subset N_{\alpha}\left(F^{j}(A)\right)
$$

for each positive integer $j$, where $\alpha=S_{2}^{j} q+\frac{\gamma}{5} \frac{S_{2}^{j+1}-1}{S_{2}-1}$. Since

$$
\alpha<S_{2}^{j}\left(q+\gamma \frac{S_{2}}{S_{2}-1}\right) \leq S_{2}^{j}(q+2 \gamma)
$$

(note $S_{2} \geq 2$ ), we have the inclusion (10).

Now, consider a continuous map $\omega:[0,1] \rightarrow G$. We must prove that $\omega$ is $\left(F^{n}, \delta\right)$-crooked. By the remark following Definition 2.2, it suffices to show that there exist two (disjoint except possibly for a common endpoint) intervals $I_{0}$ and $I_{1}$ in $[0,1]$ such that $F^{n}(\omega([0,1])) \subset N_{\delta}\left(F^{n}\left(\omega\left(I_{j}\right)\right)\right)$ for $j=0,1$. We may assume that $\operatorname{diam}\left(F^{n}(\omega([0,1]))\right) \geq 2 \delta>\gamma$, or there is nothing to prove. In what follows $\omega([0,1])$ is denoted by $\operatorname{Im} \omega$ for simplicity. There are two cases to consider.

CASE I: There exists an edge $R$ of $\mathcal{T}^{\prime}$ contained in $\operatorname{Im} \omega=\omega([0,1])$. If $\operatorname{Im} \omega$ contains no simple closed curve then $\left.\omega\right|_{\omega^{-1}(R)}$ is weakly confluent. If $\operatorname{Im} \omega$ contains a simple closed curve, let $\widetilde{G} \rightarrow G$ be the universal cover of $G, \widetilde{\mathcal{T}}$ the triangulation of $\widetilde{G}$ which is the lifting of $\mathcal{T}^{\prime}$, and $\widetilde{\omega}:[0,1] \rightarrow \widetilde{G}$ a lifting of $\omega$ to $\widetilde{G}$. If $\widetilde{R}$ is a 1 -simplex of $\widetilde{\mathcal{T}^{\prime}}$ contained in $\widetilde{\omega}([0,1])$, then $\left.\widetilde{\omega}\right|_{\widetilde{\omega}^{-1}(\widetilde{R})}$ is weakly confluent onto $\widetilde{R}$. Without loss of generality, we may suppose that $R$ is the homeomorphic projection of $\widetilde{R}$ in $G$. So $\left.\omega\right|_{\omega^{-1}(R)}$ is weakly confluent onto $R$.

As in the remark following Lemma 3.1, there exist (disjoint except possibly for a common endpoint) arcs $K_{0}$ and $K_{1}$ in $R$ such that $g(R)=g\left(K_{j}\right)$ 
for $j=0,1$. By the weak confluence of $\omega$ restricted to the preimage of $R$, there exist (disjoint except possibly for a common endpoint) arcs $I_{0}$ and $I_{1}$ in $[0,1]$ such that $\omega\left(I_{j}\right)=K_{j}$. By the proof of (2) and (3) of Lemma 3.2 with $A$ replaced by $\omega\left(I_{j}\right)$ and $f_{0}$ replaced by $F=f_{0} \circ g$ respectively, we have $F^{n}\left(\omega\left(I_{j}\right)\right)=G$ for $j=0,1$.

CASE II: $\operatorname{Im} \omega=\omega([0,1])$ contains no edge of $\mathcal{T}^{\prime}$. Here our argument is completely parallel to the one of [10, p. 1169]. We divide our consideration into two cases, Case II.a and Case II.b. A detailed argument is provided for Case IIa, while the argument for Case IIb is sketched briefly.

Let $m$ be the largest integer such that $F^{m}(\operatorname{Im} \omega)$ contains no edge of $\mathcal{T}^{\prime}$. Then there is a vertex $v$ in $\mathcal{T}^{\prime}$ such that $F^{m}(\operatorname{Im} \omega) \subset \operatorname{st}\left(v, \mathcal{T}^{\prime}\right)=$ the union of all edges of $\mathcal{T}^{\prime}$ containing $v$. Note that in this case $\operatorname{diam}\left(F^{m}(\operatorname{Im} \omega)\right)<2 \gamma / 5$.

CASE IIa: $\operatorname{diam}\left(g\left(F^{m}(\operatorname{Im} \omega)\right)\right) \geq 2 \gamma$. Note that $\left.g\left(F^{m}(\operatorname{Im} \omega)\right)\right)$ is a tree. As in Case B of Lemma 3.1, there is an edge $P$ of $\mathcal{T}^{\prime}$ which has $v$ as an endpoint such that

$$
g\left(F^{m}(\operatorname{Im} \omega)\right) \subset \operatorname{cl}\left(N_{\gamma / 5}\left(g\left(P \cap F^{m}(\operatorname{Im} \omega)\right)\right)\right) .
$$

Since $g$ is $\left(\operatorname{Id}_{P^{*}}, 2 \gamma / 5\right)$-crooked for each subinterval of $P$, there exist (disjoint except possibly for a common endpoint) intervals $I_{0}$ and $I_{1}$ in $[0,1]$ such that

$$
g\left(F^{m}(\operatorname{Im} \omega) \cap P\right) \subset \operatorname{cl}\left(N_{2 \gamma / 5}\left(g\left(F^{m}\left(\omega\left(I_{j}\right)\right)\right)\right)\right) \quad \text { for } j=0,1 .
$$

Hence,

$$
\begin{aligned}
& g\left(F^{m}(\operatorname{Im} \omega)\right) \subset \operatorname{cl}\left(N_{\gamma / 5}(g(K))\right) \subset \operatorname{cl}\left(N_{3 \gamma / 5}\left(g\left(F^{m}\left(\omega\left(I_{j}\right)\right)\right)\right)\right) \\
& \text { for } j=0,1 .
\end{aligned}
$$

So $g$ is $\left(F^{m}, 3 \gamma / 5\right)$-crooked. Now

$$
\begin{aligned}
F^{m+1}(\operatorname{Im} \omega) & =h\left(g\left(F^{m}(\operatorname{Im} \omega)\right)\right) \\
& \subset h\left(\operatorname{cl}\left(N_{3 \gamma / 5}\left(g\left(F^{m}\left(\omega\left(I_{j}\right)\right)\right)\right)\right), \quad j=0,1, \text { by }(11)\right. \\
& \subset \operatorname{cl}\left(N_{S_{2} 3 \gamma / 5}\left(h\left(g\left(F^{m}\left(\omega\left(I_{j}\right)\right)\right)\right)\right)\right) \quad \text { by }(5) \\
& \subset N_{S_{2} \gamma}\left(F^{m+1}\left(\omega\left(I_{j}\right)\right)\right), \quad j=0,1 .
\end{aligned}
$$

As $\operatorname{diam}\left(g\left(F^{m}(\operatorname{Im} \omega)\right)\right) \geq 2 \gamma$, by (11) we have $\operatorname{diam}\left(g\left(F^{m}\left(\omega\left(I_{j}\right)\right)\right)\right) \geq 4 \gamma / 5$. By $(9), \operatorname{diam}\left(F^{m+1}\left(\omega\left(I_{j}\right)\right)\right) \geq 2 \min \left\{\operatorname{diam}\left(g\left(F^{m}\left(\omega\left(I_{j}\right)\right)\right)\right), \gamma / 5\right\}=2 \gamma / 5$ $>\gamma / 5$ for $j=0,1$. Now using (11), (5), (9), (10), (8) and (6), one can show that

$$
\begin{aligned}
F^{n}(\operatorname{Im} \omega) & \subset F^{n-m-1}\left(N_{S_{2} \gamma}\left(F^{m+1}\left(\omega\left(I_{j}\right)\right)\right)\right) \\
& \subset N_{S_{2}^{n-m-1}\left(S_{2} \gamma+2 \gamma\right)}\left(F^{n}\left(\omega\left(I_{j}\right)\right)\right) \quad \text { by }(9) \text { and }(10) \\
& \subset N_{\delta}\left(F^{n}\left(\omega\left(I_{j}\right)\right)\right) \quad \text { by }(8) \text { and }(6), \text { for } j=0,1 .
\end{aligned}
$$

Hence $\omega$ is $\left(F^{n}, \delta\right)$-crooked. 
CASE II.b: $\operatorname{diam}\left(g\left(F^{m}(\operatorname{Im} \omega)\right)\right)<2 \gamma$. By the choice of $m, F^{m+1}(\operatorname{Im} \omega)$ contains an edge $R$ of $\mathcal{T}^{\prime}$. This together with (5) yields

$$
\gamma / 5 \leq \operatorname{diam}\left(F^{m+1}(\operatorname{Im} \omega)\right) \leq 2 S_{2} \gamma<\varepsilon \quad \text { by }(8) .
$$

The above inequality means that $F^{m+1}(\operatorname{Im} \omega)$ contains at least a half of an edge of $\mathcal{T}^{\prime}$, but still sits in a tree with at most one branch point. Then one can proceed as in Case II.a to conclude that $\omega$ is $\left(F^{n}, \delta\right)$-crooked as well.

This proves (5), and hence completes the proof.

Proof of Main Theorem. Suppose that a map $f: G \rightarrow G$ satisfies condition $(*)$ in the Main Theorem and an $\varepsilon>0$ is given. First, using Lemma 3.2 , we construct a map $f_{0}$ satisfying $(*)$ and $(+)_{\beta}$, where $f_{0}$ is $\varepsilon / 2$-close to $f$. Then as in [10], Lemma 3.3 enables us to construct a sequence $\left\{f_{i}: G \rightarrow G \mid i \geq 0\right\}$ of surjections and an increasing sequence $\{n(i) \mid i \geq 0\}$ of integers such that

(a) $n(0)=0$.

(b) $d\left(f_{i}, f_{i+1}\right)<\varepsilon / 2^{i+1}$.

(c) Each map $\omega:[0,1] \rightarrow G$ is $\left(f_{i}^{n(k)},\left(\varepsilon / 2^{k}-\varepsilon / 2^{k+i}\right)\right)$-crooked for each $k=1, \ldots, i+1$.

(d) For each subcontinuum $A$ of $G$ with $\operatorname{diam}(A)>\varepsilon / 2^{k}, f_{i}^{n(k)}(A)=G$ for each $k=0,1, \ldots, i$.

By (b), the limit map $f_{\varepsilon}=\lim _{i \rightarrow \infty} f_{i}$ exists and $f_{\varepsilon}$ is $\varepsilon / 2$-close to $f_{0}$. Since $f_{0}$ is $\varepsilon / 2$-close to $f, f_{\varepsilon}$ is $\varepsilon$-close to $f$. Condition (c) and Propositions 2.5 and 2.6 guarantee that $\left(G, f_{\varepsilon}\right)$ is hereditarily indecomposable. This completes the proof of the Main Theorem.

4. Applications. For every integer $p \geq 1$, there exists a topolotgically unique hereditarily indecomposable circle-like continuum $\Sigma(p)=$ $\lim _{\leftarrow}\left(S_{i}, f_{i}: S_{i+1} \rightarrow S_{i}\right)$, where each $S_{i}$ is a simple closed curve and $\operatorname{deg} f_{i}=p$ ([5] and [11]), and it is called the pseudo-solenoid of type $p$ if $p>1$ and the pseudo-circle if $p=1$. The $p$-adic solenoid is denoted by $S(p)(S(1)$ is the simple closed curve).

We assume that each simple closed curve under consideration is the unit circle in the complex plane, i.e. $S^{1}=\{z \in \mathbb{C}|| z \mid=1\}$. The map $f_{p}: S^{1} \rightarrow S^{1}$ defined by $f_{p}(z)=z^{p}$ gives the $p$-adic solenoid as its inverse limit $\left(S^{1}, f_{p}\right)$. If $p>1$, it is easy to see that $f_{p}$ satisfies the conditions of the Main Theorem. If $p=1$, it is easy to construct a map $\phi_{1}: S^{1} \rightarrow S^{1}$ of degree 1 satisfying the condition of the Main Theorem. Applying the Main Theorem to $f_{p}$ and $\phi_{1}$ respectively (with the help of the uniqueness of $\Sigma(p)$ ), we have the following inverse sequence representation of $\Sigma(p)$ by a single 
bonding map. The authors are grateful to Professor P. Minc for pointing out the $p=1$ case.

Corollary 4.1. The pseudo-solenoid or pseudo-circle $\Sigma(p)(p \geq 1)$ can be represented as an inverse limit $\left(S^{1}, g_{p}\right)$ of a map $g_{p}: S^{1} \rightarrow S^{1}$ of the simple closed curve with degree $p$.

Suppose that $g_{p}: S^{1} \rightarrow S^{1}$ is a map as in the above corollary. By the homotopy lifting property of $f_{p}$, it is easy to construct a sequence $\left\{h_{i}: S^{1} \rightarrow S^{1}\right\}$ of surjections such that $h_{i} \circ g_{p}=f_{p} \circ h_{i+1}$. The sequence induces a surjective map $h_{\infty}=\lim _{\leftarrow}\left(h_{i}\right): \Sigma(p) \rightarrow S(p)$. Applying Proposition B of [7], we can choose $\left(h_{i}\right)$ so that $h_{i}=h_{i+1}$ for each $i$. The next corollary gives a little more information on these maps.

Corollary 4.2. Suppose that $p>1$. For each $\varepsilon>0$, there exist surjections $g, h: S^{1} \rightarrow S^{1}$ such that

(1) the inverse limit $\left(S^{1}, g\right)$ is homeomorphic to $\Sigma(p)$,

(2) $h \circ g=f_{p} \circ h$ and hence $h$ induces a surjection $h_{\infty}: \Sigma(p) \rightarrow S(p)$,

(3) $\varrho(h$, id $)<\varepsilon$.

Proof. Since $f_{p}$ is a positively expansive open map, it has the following property called the pseudo-orbit tracing property: for each $\varepsilon>0$, there is a $\delta>0$ such that for each sequence $\left\{x_{i} \mid i \geq 0\right\}$ of points of $S^{1}$ with $\varrho\left(f_{p}\left(x_{i}\right), x_{i+1}\right)<\delta$ for each $i \geq 0$, there exists a point $x$ of $S^{1}$ such that $\varrho\left(f_{p}^{i}(x), x_{i}\right)<\varepsilon$ for each $i$.

Apply the Main Theorem to obtain a map $g: S^{1} \rightarrow S^{1}$ such that $\varrho\left(f_{p}, g\right)<\delta$ and $\left(S^{1}, g\right)$ is hereditarily indecomposable, and hence, is the pseudo-solenoid of type $p$. For each $x$ in $S^{1}$, consider the sequence $\left\{g^{i}(x) \mid\right.$ $i \geq 0\}$. Then $\varrho\left(f\left(g^{i}(x)\right), g^{i+1}(x)\right)<\delta$ for each $i \geq 0$, and the pseudo-orbit tracing property guarantees the existence of a point $h(x)$ of $S^{1}$ such that $\varrho\left(f_{p}^{i}(h(x)), g^{i}(x)\right)<\varepsilon$ for each $i \geq 0$. The remaining part of the proof proceeds as those of the topological stability of expansive homeomorphisms with the pseudo-tracing property (see for example, [1, Theorem 5.8, p. 684]).

Remark. For a connected graph $G, \operatorname{Id}_{G}$ does not satisfy condition $(*)$. However, as in the above argument for the case $p=1$, the construction of Lemma 3.1 yields a piecewise linear map approximating $\operatorname{Id}_{G}$ and satisfying (*). Thus $\operatorname{Id}_{G}$ can be approximated by a map $h: G \rightarrow G$ such that $(G, h)$ is hereditarily indecomposable.

\section{References}

[1] N. Aoki, Topological Dynamics, Chap. 15 in: Topics in General Topology, K. Morita and J. Nagata (eds.), North-Holland, 1989, 625-740. 
[2] M. Barge and B. Diamond, The dynamics of continuous maps of finite graphs through inverse limits, Trans. Amer. Math. Soc. 344 (1994), 773-790.

[3] R. H. Bing, A homegeneous indecomposable plane continuum, Duke Math. J. 15 (1948), 729-742.

[4] L. Block, J. Keesling and V. V. Uspenskij, Inverse limits which are the pseudoarc, Houston J. Math. 26 (2000), 629-638.

[5] L. Fearnley, The classification of all hereditarily indecomposable circularly chainable continua, Trans. Amer. Math. Soc. 168 (1972), 387-401.

[6] G. W. Henderson, The pseudo-arc as an inverse limit with one binding map, Duke Math. J. 31 (1964), 421-425.

[7] K. Hiraide, Positively expansive open maps on Peano spaces, Topology Appl. 37 (1990), 213-220.

[8] D. P. Kuykendall, Irreducibility and indecomposability in inverse limits, Fund. Math. 80 (1973), 265-270.

[9] P. Minc, A hereditarily indecomposable tree-like continuum without the fixed point property, Trans. Amer. Math. Soc. 352 (1999), 643-655.

[10] P. Minc and W. R. R. Transue, A transitive map on $[0,1]$ whose inverse limit is the pseudo-arc, Proc. Amer. Math. Soc. 111 (1991), 1165-1170.

[11] J. T. Rogers, Jr., Pseudo-circles and universal circularly chainable continua, Illinois J. Math. 14 (1970), 222-237.

[12] R. F. Williams, One-dimensional non-wandering sets, Topology 6 (1987), 473-483.

[13] - Classification of one-dimensional attractors, in: Global Analysis (Berkeley, CA, 1968), Proc. Sympos. Pure Math. 14, Amer. Math. Soc., 1970, 341-361.

Institute of Mathematics

University of Tsukuba

Tsukuba, Ibaraki 305-8571, Japan

E-mail: kawamura@math.tsukuba.ac.jp

Department of Mathematics and Statistics

University of Saskatchewan

106 Wiggins Road

Saskatoon, Saskatchewan

Canada S7N 5E6

E-mail: tymchat@math.usask.ca
Faculty of Arts and Science

Nipissing University

100 College Drive, Box 5002

North Bay, Ontario

Canada P1B 8L7

E-mail: muratt@numail.nipissingu.ca

Received 2 December 2002;

in revised form 20 January 2005 